\title{
The mTOR Pathway Is Activated in Human Autosomal-Recessive Polycystic Kidney Disease
}

\author{
Jan Ulrich Becker ${ }^{b, d} \quad$ Anabelle Opazo Saez ${ }^{a} \quad$ Klaus Zerres $^{\text {e }}$ Oliver Witzke ${ }^{a}$ \\ Peter Friedrich Hoyer ${ }^{c}$ Kurt Werner Schmid ${ }^{b}$ Andreas Kribben ${ }^{a}$ \\ Carsten Bergmann $^{e, f}$ Jens Nürnberger ${ }^{a}$ \\ ${ }^{a}$ Department of Nephrology, ${ }^{b}$ Institute of Pathology and Neuropathology, and ' Clinic of Pediatric Nephrology, \\ University Hospital of Essen, University Duisburg-Essen, Essen, ${ }^{d}$ Department of Pathology, Hannover Medical \\ School, Hannover, ${ }^{e}$ Department of Human Genetics, Aachen University, Aachen, and ${ }^{\mathrm{f} B i o s c i e n t i a}$ Center for Human \\ Genetics, Ingelheim, Germany
}

\section{Key Words}

Cystic kidney disease $\cdot$ Autosomal-recessive polycystic kidney disease $\cdot \mathrm{mTOR}$

\begin{abstract}
Background: An inappropriate activation of the mTOR pathway was demonstrated in the autosomal dominant (AD) form of polycystic kidney disease (PKD). To date it is unclear whether the mTOR pathway is activated in autosomal-recessive (AR) PKD, a cystic disease which occurs in childhood. The purpose of the present study was to evaluate the mTOR pathway in AR PKD. Methods: We evaluated the expression of mTOR pathway molecules in paraffin-embedded liver and kidney samples from patients with AR PKD and control specimens from animals as well as humans. Monoclonal antibodies, the phosphorylated proteins pmTOR, pS6-ribosomal-protein (pS6K), p4E-BP1, pelF4G, and phospho-tuberin/TSC2 were used. $\boldsymbol{R} \boldsymbol{e}$ sults: mTOR was strongly expressed in renal cyst-lining cells and bile ducts from AR PKD specimen. S6K immunostaining was strong in smaller tubules and weak both in larger renal
\end{abstract}

cysts and in the bile duct epithelium. In controls, mTOR and S6K were expressed in distal tubule segments. 4E-BP1-immunostaining was restricted to noncystic tubules in AR PKD. elFG4-immunostaining was observed in bile duct epithelium in AR PKD, but not in control tissue. Tuberin/TSC2 immunostaining was negative in all specimens. Conclusion: Our data suggest that the mTOR pathway may be activated in AR PKD, and mTOR molecules may represent a potential target to slow down cyst development in this disease.

Copyright $\odot 2010$ S. Karger AG, Basel

\section{Introduction}

Polycystic kidney diseases (PKD) include various disorders characterized by cystic kidneys and variable multiorgan pathology [1]. Autosomal-recessive (AR) PKD is a form of childhood PKD which is characterized by rapidly progressive cyst development and congenital hepatic fibrosis (CHF). CHF is characterized by bridging hepatic fibrosis and proliferation of irregularly shaped small in-

\section{KARGER}

Fax +41613061234 E-Mail karger@karger.ch www.karger.com
(C) 2010 S. Karger AG, Basel

$1420-4096 / 10 / 0332-0129 \$ 26.00 / 0$

Accessible online at:

www.karger.com/kbr
Jens Nürnberger

Department of Nephrology, University Hospital of Essen

Hufelandstrasse 55

DE-45122 Essen (Germany)

Tel. +49 201723 3955, Fax +49 201723 3855, E-Mail jens.nuernberger@ uni-due.de 
trahepatic bile ducts. The latter can be considered the hepatic equivalent to renal cyst development.

Advances in the understanding of the molecular basis for PKD have led to promising new therapeutic approaches to slow down progression of cyst development [2]. One such strategy includes inhibition of the mammalian target of rapamycin (mTOR), a conserved Ser/Thr kinase that regulates various cellular processes including growth and metabolism [3,4]. Recently, an activation of the mTOR pathway has been described in cystic tubules in mouse models and in the human autosomal dominant (AD) form of PKD [5].

mTOR is a member of the phosphoinositide kinaserelated kinase (PIKK) family that phosphorylates proteins on serine or threonine residues [6]. Target molecules of mTOR include ribosomal S6 kinase and eukaryote initiation factor $4 \mathrm{E}$ binding protein 1 , key translation regulators for protein synthesis. mTOR activation is regulated by a variety of signaling pathways including small GTPases, Rheb, akt, Raptor, and the TSC1-TSC2 complex [3]. Recently, Wahl et al. [7] demonstrated an enhanced phosphorylation of akt, an upstream regulator of mTOR, in Han:SPRD rats.

To date, several experimental studies in rodent models of PKD have revealed that inhibition of the MTOR pathway results in reduction of kidney size, prevents the loss of kidney function, and lowers cyst volume [5, 8-10]. Furthermore, retrospective observations from patients with $\mathrm{PKD}$ and end-stage renal disease who underwent kidney transplantation have shown that treatment with sirolimus, an mTOR inhibitor, reduced kidney volumes by $25 \%$ [5]. Currently, several clinical trials have started to investigate the effect of mTOR inhibitors in patients with AD PKD [2].

Fischer et al. [11] have reported an activation of the mTOR pathway in kidneys from patients with AR PKD. In the present study, we investigated the mTOR pathway in kidney and liver specimens from patients with AR PKD. Furthermore, we evaluated the physiological renal and hepatic expression of these molecules in animals as well as humans. Shillingford et al. [5] reported an inappropriate activation of the mTOR pathway in the autosomal dominant form of PKD using antibodies against phosphorylated, active forms of mTOR (pmTOR) and its downstream effector S6 kinase (pS6K). In addition, mTOR-inhibition has been shown to slow down cyst formation in several rodent models of PKD [12]. In our study, we have focused our analysis on phosphorylated forms of the mTOR-related molecules. We analyzed expression of the upstream mTOR-regulator tuberin, and the mTOR effectors S6K, eIF4G, and 4E-BP1.

\section{Materials and Methods}

Reagents, Antibodies, and Animals

Rabbit-monoclonal antibodies against the following phosphorylated, activated forms of kinases of the mTOR pathway were purchased from Cell Signaling Technology, Frankfurt am Main, Germany: pmTOR (Ser2448), pS6K (Ser235/236), p4E-BP1 (Thr37/46), peIF4G (Ser1108), and p-tuberin/TSC2 (Thr1462). All other chemicals and reagents were supplied by Sigma. BALB/c mice and Sprague-Dawley rats (Charles River, Sulzfeld, Germany) were used for experiments.

\section{Specimen}

Human liver and kidney specimens from patients with AR PKD were obtained from subjects who underwent either combined kidney - liver transplantation or received grafts consecutively. Human control kidneys were obtained from patients who underwent complete unilateral nephrectomy or hepatectomy due to carcinoma. Sections were taken from regions morphologically free from any signs of cancer. Rodent kidneys were fixed by perfusion with $4 \%$ paraformaldehyde in PBS, and subsequently embedded in paraffin [13]. The study was approved by the local Ethics Committee of the University Hospital Essen.

\section{Immunostaining}

Immunostaining was carried out on 4 - $\mu \mathrm{m}$-thick paraffin-wax sections. Antigen retrieval was carried out with $0.01 \mathrm{M}$ citrate buffer at $\mathrm{pH} 6.0$ for $25 \mathrm{~min}$ in a hot water bath $\left(95^{\circ} \mathrm{C}\right)$.

Primary antibodies were incubated for $60 \mathrm{~min}$ and antibodies were demonstrated with a commercially available antibody antirabbit IgG detection kit (ZytoChem-Plus HRP Polymer-Kit, Zytomed Systems, Berlin, Germany). Sections were counterstained with Mayer's hemalum solution (Merck, Darmstadt, Germany). Sections were coverslipped and examined with a Zeiss Axio Imager equipped with an AxioCam MRC Camera (Carl Zeiss Jena GmbH, Jena, Germany).

Patients

Table 1 displays the characteristics of the cohort. Mutation screening was done for the 66 exons encoding the 4074 aa polyductin protein (GenBank NM_138694) by denaturing high-performance liquid chromatography (DHPLC) on a Wave Fragment Analysis System (Transgenomic, Crewe, UK), as recently described in detail [14].

\section{Results}

Patients were between 4 and 14 years old when they received a liver transplant (table 1). In 2 patients, two mutations in the PKHD1 gene were detected; in another 2 patients only one mutation in the PKHD1 gene was detected, and in another 2 patients no mutation in the PKDH1 gene was found. All detected missense mutations affect evolutionarily conserved residues of the polyductin/fibrocystin protein and were absent among 200 ethnically matched controls. Specimens from all 6 
Table 1. Characteristics of study cohort

\begin{tabular}{|c|c|c|c|c|c|c|}
\hline $\begin{array}{l}\text { Patient } \\
\text { No. }\end{array}$ & Sex & Age at transplantation & $\begin{array}{l}\text { Age at diag- } \\
\text { nosis, years }\end{array}$ & $\begin{array}{l}\text { Age } \\
\text { at ESRF }\end{array}$ & $\begin{array}{l}\text { PKHD1 } \\
\text { mutations }\end{array}$ & Figure \\
\hline 1 & M & combined kidney-liver transplantation at 12 years & 1 & 7 & $\begin{array}{l}\text { c. } 4457 \mathrm{C}>\mathrm{T}(\mathrm{p} . \mathrm{P} 1486 \mathrm{~L})(\mathrm{M}) \\
\text { c. } 9464 \mathrm{~A}>\mathrm{G}(\mathrm{p} . \mathrm{Y} 3155 \mathrm{C})(\mathrm{P})\end{array}$ & $2 c, d$ \\
\hline 2 & M & combined kidney-liver transplantation at 7 years & 2 & 7 & $\begin{array}{l}\text { c.107C > T (p.T36M) (M) } \\
\text { c.10219C>T (p.Q3407X) (P) }\end{array}$ & $1 \mathrm{a}, 1 \mathrm{~b}, 3 \mathrm{c}, 3 \mathrm{~d}$ \\
\hline 3 & $\mathrm{~F}$ & combined kidney-liver transplantation at 4 years & 1 & 4 & no mutation detected & $3 a, 3 b$ \\
\hline 4 & $\mathrm{M}$ & $\begin{array}{l}\text { kidney transplantation with } 5 \text { years } \\
\text { liver transplantation at } 6 \text { years }\end{array}$ & 1 & 4 & c.5895dupA (p.L1966fs) & $\begin{array}{l}1 c, 1 d, 2 a \\
2 b, 4 e, 4 f\end{array}$ \\
\hline 5 & M & combined kidney-liver transplantation at 13 years & 7 & 13 & no mutation detected & $2 e, f$ \\
\hline 6 & $\mathrm{~F}$ & combined kidney-liver transplantation at 14 years & 8 & 12 & c.9767C>T (p.P3256L) & $4 c, 4 d$ \\
\hline
\end{tabular}

patients were analyzed; the presented specimens are denoted in table 1, the specimens studied are shown in table 1.

\section{Expression of pmTOR}

Antibodies against phosphorylated molecules were used to evaluate the activation of the mTOR pathway.

Figure 1 shows the distribution of pmTOR in human kidneys with AR PKD and human and murine control kidneys. In AR PKD kidneys, pmTOR was strongly expressed in cyst lining cells whereas there was no expression or very low levels of expression detectable in noncystic tubules with the exception of a few tubule cells. The subcellular staining pattern was characterized by an intracellular granular distribution with varying intensity between tubule cells. In control kidneys, there was a clearly positive signal in distal tubules and collecting ducts, whereas proximal tubuli and glomeruli were negative. This staining pattern was observed in human (fig. 1e, f), murine (fig. 1g, h) and rat kidneys (data not shown).

Figure 2 shows the expression of pmTOR in human liver specimen from patients with and without AR PKD. Three liver explants from patients with AR PKD are shown to illustrate the variety of the bile duct morphology in AR PKD ranging from few massively enlarged and elongated bile ducts (fig. 2a, b) to small and irregularly shaped bile ducts (fig. 2e, f). pmTOR immunostaining was strongly positive in all bile ducts in livers from patients with AR PKD. Bile duct cells showed a strong ubiquitous intracy toplasmic staining pattern. In contrast, bile ducts from human control livers showed only a weak immunostaining for pmTOR restricted to the apical cell border (fig. 2g, h).

\section{Distribution of Phosphorylated Ribosomal Protein S6 (pS6K)}

Figure 3 shows the distribution of pS6K in human kidneys with AR PKD and control kidneys from humans and mice.

The immunostaining showed a heterogeneous distribution with strong positive staining in smaller tubules compared to the pmTOR staining in AR PKD kidneys. Larger cyst exhibited only a weak positive signal for pS6K, in some cystic tubules the signal was even completely absent. Positive staining appeared to involve all cells from one affected tubule. In control kidneys, pS6K immunostaining was restricted to the thin ascending part of the loop of Henle. In addition, there was a weaker staining signal of distal tubules in human (fig. 3e, f) and murine kidney (fig. 3g, h; data not shown for rats).

Figure 4 shows the expression of pS6K in human liver specimen from patients with and without AR PKD. Samples from three AR PKD livers are shown in figure $4 a-f$. pS6K immunostaining was most intense in parenchymal hepatic cells. Bile ducts also exhibited a positive but weaker signal for pS6K labeling. In contrast, bile ducts from controls were completely negative for pS6K (fig. 4g, h).

\section{Distribution of $p 4 E-B P 1$ and peIFG4}

Figure 5 shows the distribution of p4E-BP1 and peIF4G in AR PKD kidneys, livers and controls. p4E- 
Fig. 1. Renal expression of activated mTOR. The left panel shows lower magnifications at $10 \times$, the right higher magnifications at $40 \times$ of the same specimen. a-d pmTOR expression in 2 different human AR PKD kidneys. Strong labeling is observed in epithelial cyst lining cells whereas non-cystic tubules (proximal tubule, PT) were negative. e, $\mathbf{f}$ pmTOR expression in a human control kidney. $\mathbf{g}, \mathbf{h}$ pmTOR expression in a murine kidney. In both, pmTOR expression was clearly detectable in distal tubules (DT), the convoluted segments of distal tubules (DCT), and collecting ducts (CD), whereas proximal tubules (PT) and glomeruli (GL) were negative. The same immunostaining pattern was observed in murine kidney sections $(\mathbf{g}, \mathbf{h})$.
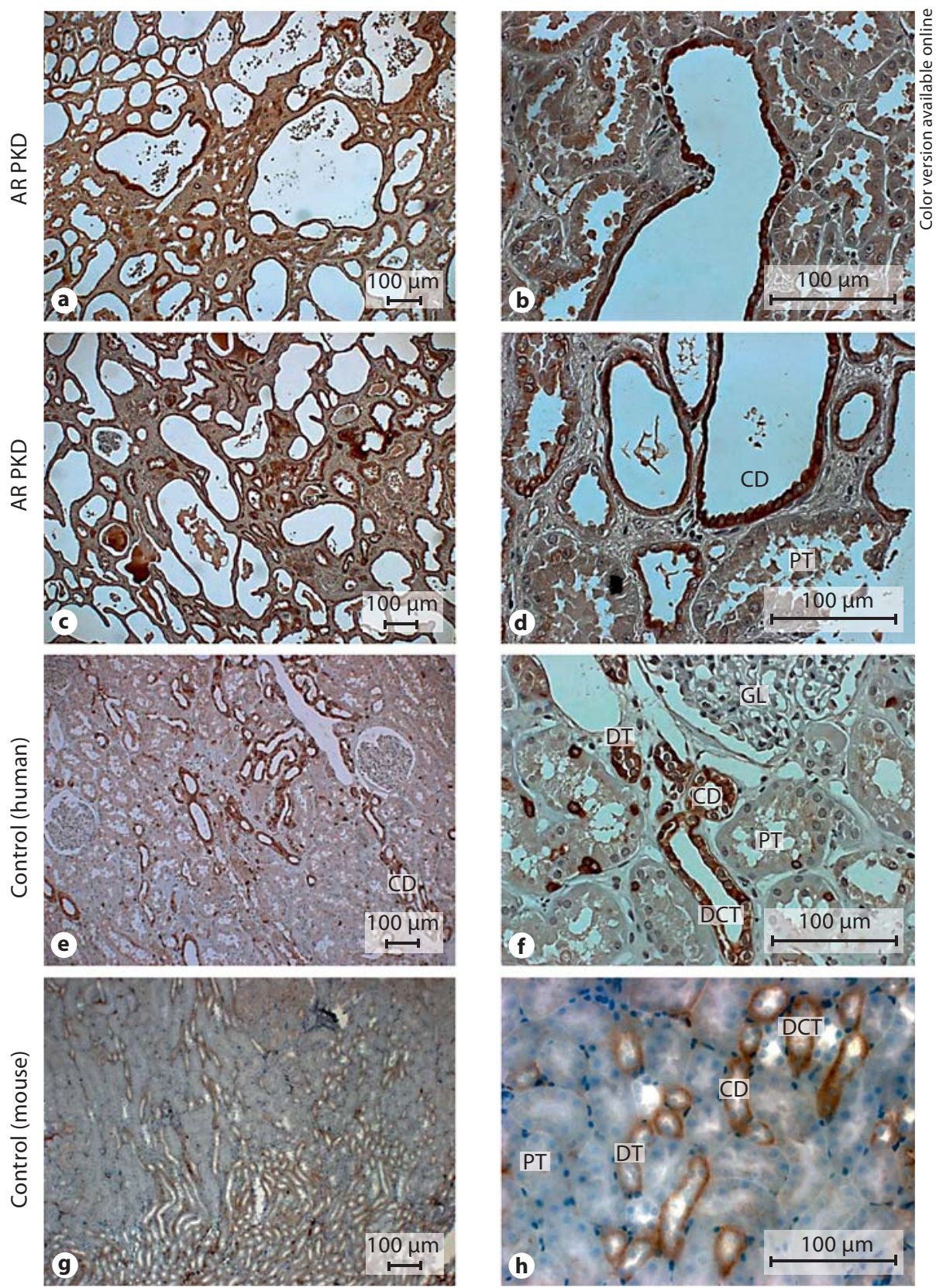

BP1 immunostaining was restricted to noncystic tubules in AR PKD, but there was no positive signal in cyst lining tubule cells. In the control kidney, few (distal convoluted) tubules were slightly positive (fig. $5 \mathrm{c}, \mathrm{d}$ ). In the liver, no p4E-BP1 labeling could be detected, neither in AR PKD (fig. 5e, f) nor in controls (data not shown).
AR PKD kidneys and control kidneys were negative for peIF4G immunostaining (data not shown). In contrast, in specimens from AR PKD livers, peIF4G immunostaining was observed in the bile duct epithelium (fig. $5 \mathrm{~g}, \mathrm{~h}$ ), whereas peIF4G immunostaining in control liver was negative. $\mathrm{p}$-Tuberin/TSC2 immunostaining was negative in all kidney and liver specimens. 
mTOR
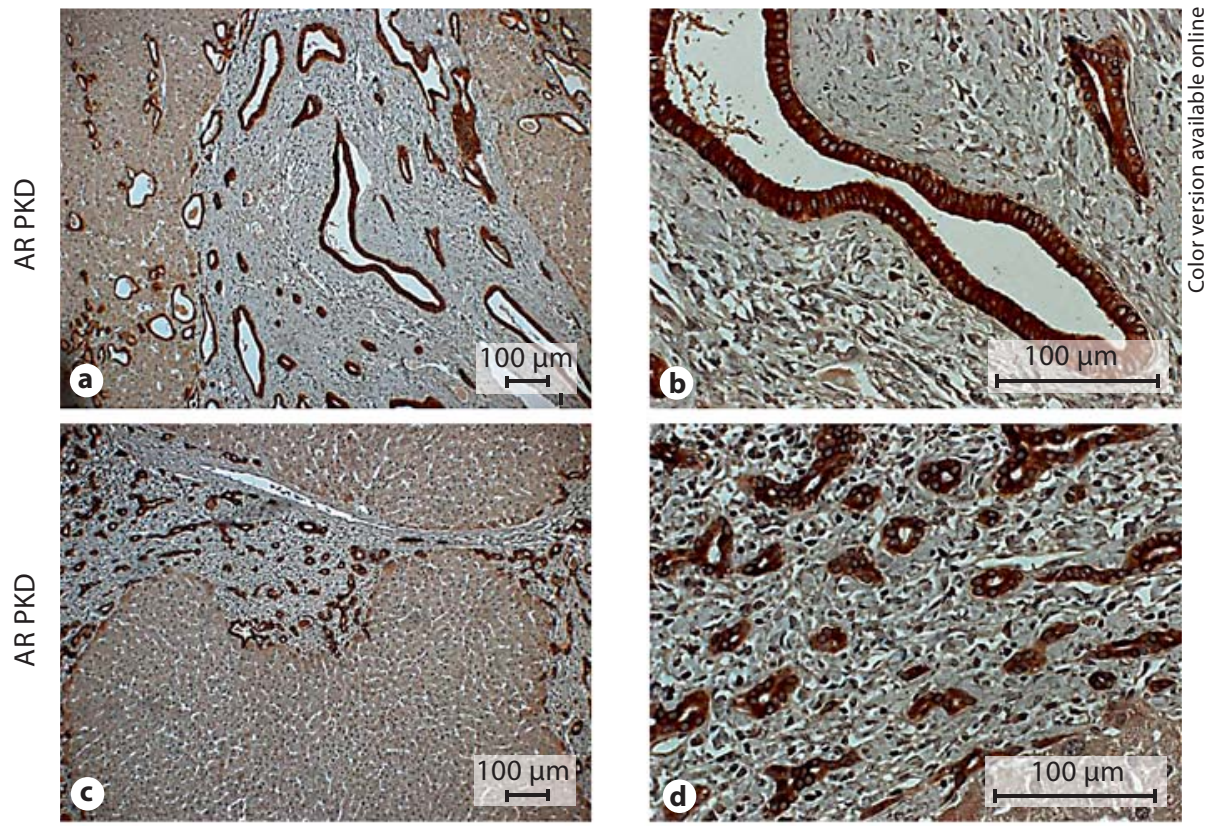

Fig. 2. Hepatic expression of activated pmTOR. a, c, e Lower magnifications at $10 \times$. b, d, f Higher magnifications at $40 \times$ of the same liver specimens (except for controls $\mathbf{g}$ and $\mathbf{h}$ ). a-f pmTOR expression in 3 different human AR PKD livers. Bile ducts of AR PKD patients (a-f) exhibited a strong immunostaining. $\mathbf{g}, \mathbf{h}$ pmTOR expression in two human control livers at higher magnifications at $40 \times$. In these specimens, a weak immunostaining for pmTOR was only detected at the apical border of the bile ducts (insets show further magnifications of bile ducts).
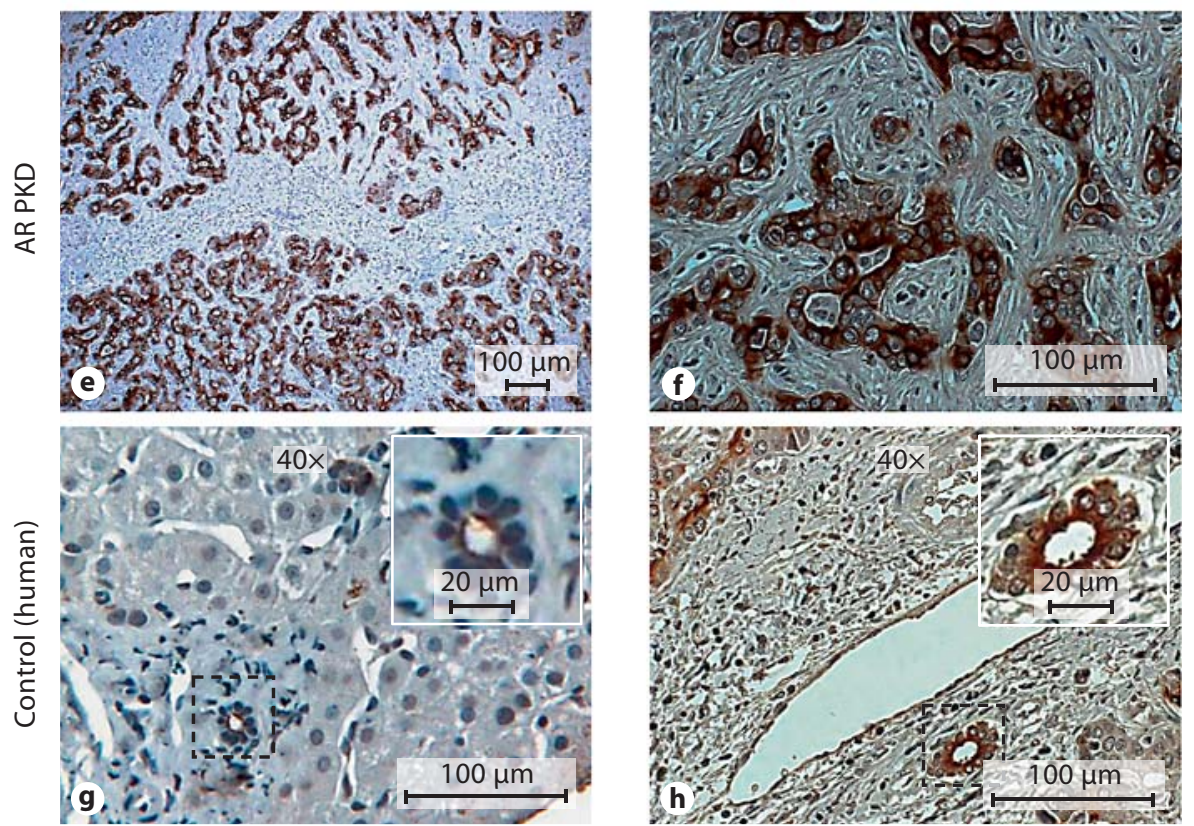

To preclude an impact of age on the expression profile of the analyzed molecules, experiments were performed using specimens from juvenile human controls (8-13 years) and murine pups. There was no difference in the immunostaining pattern between young and adult controls.

\section{Discussion}

In the present study, we evaluated whether the mTOR pathway is activated in human AR PKD. We found that molecules of the mTOR pathway were strongly expressed in cyst lining renal epithelial cells and the bile duct epithelium. 
S6K
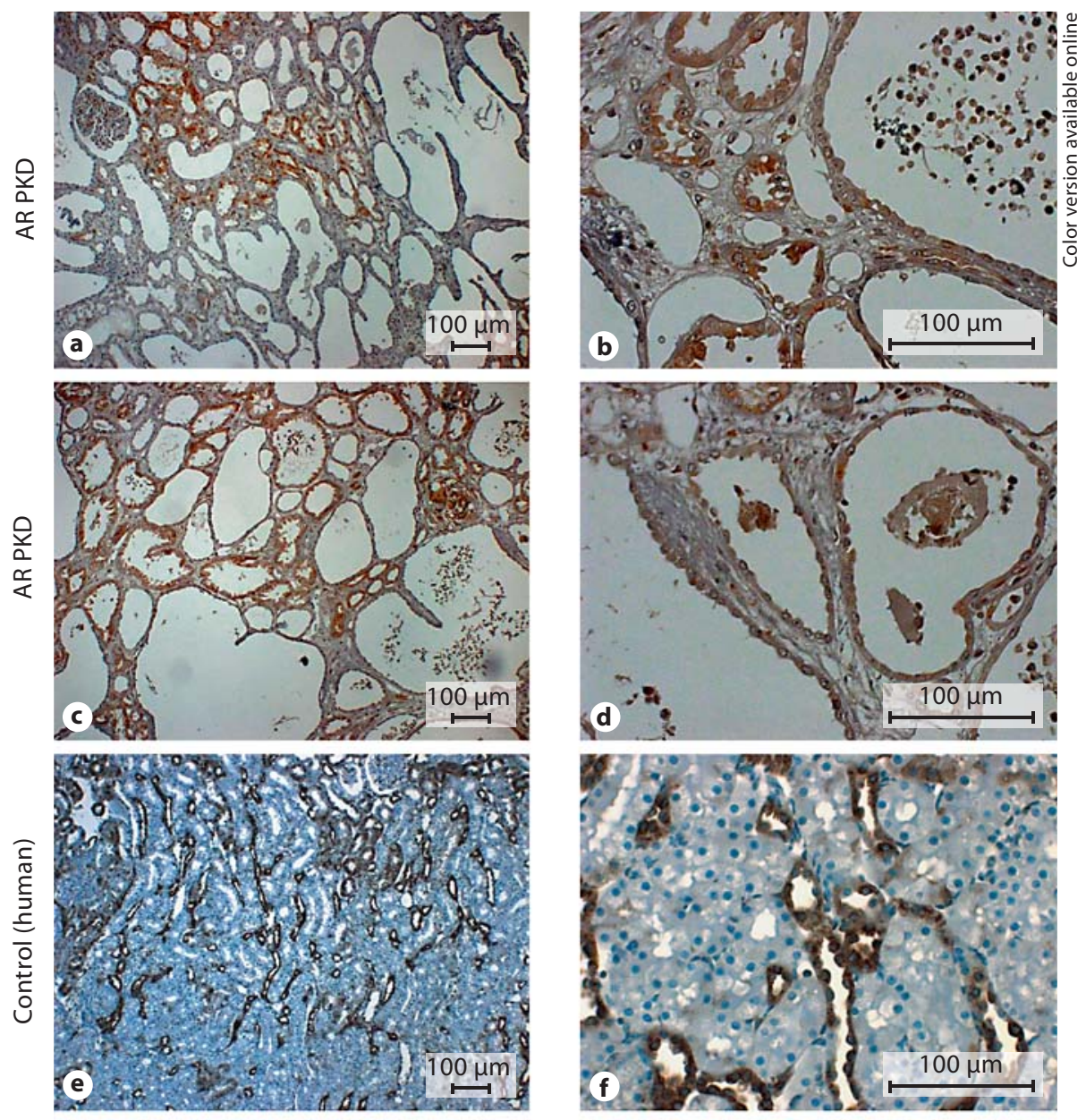

Fig. 3. Renal expression of activated ribosomal pS6K. The left panel shows lower magnifications at $10 \times$, the right higher magnifications at $40 \times$ of the same specimen. a-d pS6K expression in 2 different human AR PKD kidneys. pS6K immunostaining is observed in noncystic tubules, whereas cyst lining tubule cells exhibited a weak staining signal at the most. e, f pS6K expression in a human control kidney. $\mathbf{g}$, $\mathbf{h}$ pS6K expression in a murine kidney. In both, pS6K expression was clearly detectable in distal tubules, whereas proximal tubules and glomeruli were negative. The same immunostaining pattern was observed in murine kidney sections $(\mathbf{g}, \mathbf{h})$.
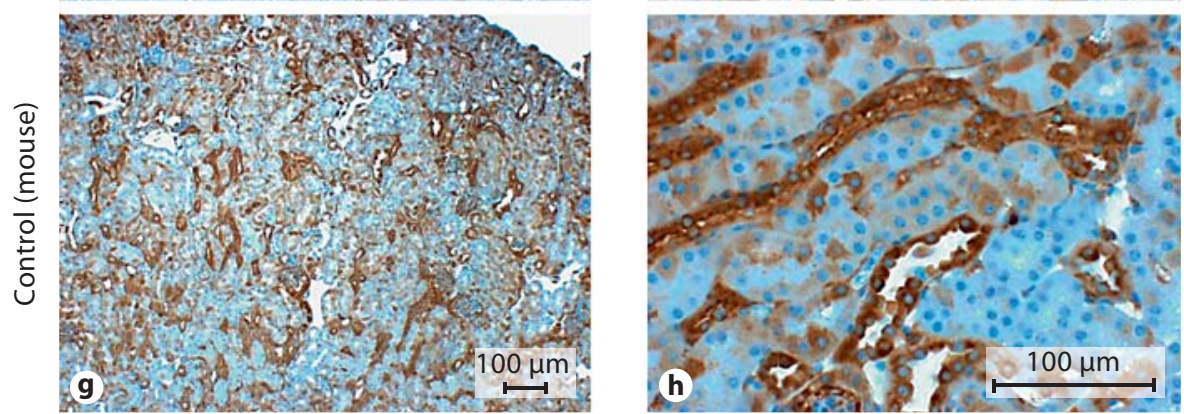

These data suggest that mTOR pathway activity may be related to the cystic changes found in AR PKD. It is possible that mTOR pathway may participate in the cyst development in renal tubules and bile ducts in patients with AR PKD and in the $b p k$ mouse, a murine model of early-onset AR PKD, mTOR inhibition significantly improved the cystic phenotype [5]. In the current study, we provide additional evidence for an activation of the mTOR pathway in human AR PKD. mTOR inhibitors are already used in children after solid organ transplantation, and may also represent a suitable therapeutic target to slow down cyst progression in human AR PKD.

We observed different labeling patterns for pmTOR and pS6K immunostaining in AR PKD kidneys. pS6K is 
S6K
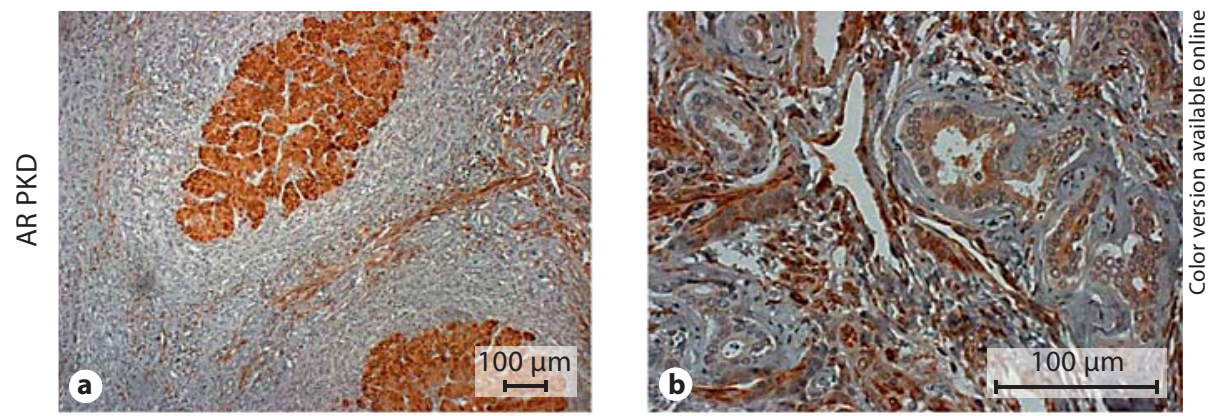

Fig. 4. Hepatic expression of activated ribosomal pS6K. a, c, e Lower magnifications at $10 \times . \mathbf{b}, \mathbf{d}, \mathbf{f}$ Higher magnifications at $40 \times$ of the same liver specimens (except for controls $\mathbf{g}$ and $\mathbf{h}$ ). a-f pS6K expression in 3 different human AR PKD livers. Bile ducts of AR PKD patients (a-f) exhibited a positive immunostaining. $\mathbf{g}, \mathbf{h}$ pS6K expression in two human control livers at higher magnifications at $40 \times$. In these specimens, no immunostaining for $\mathrm{pS} 6 \mathrm{~K}$ was in bile ducts (insets show further magnifications of bile ducts).
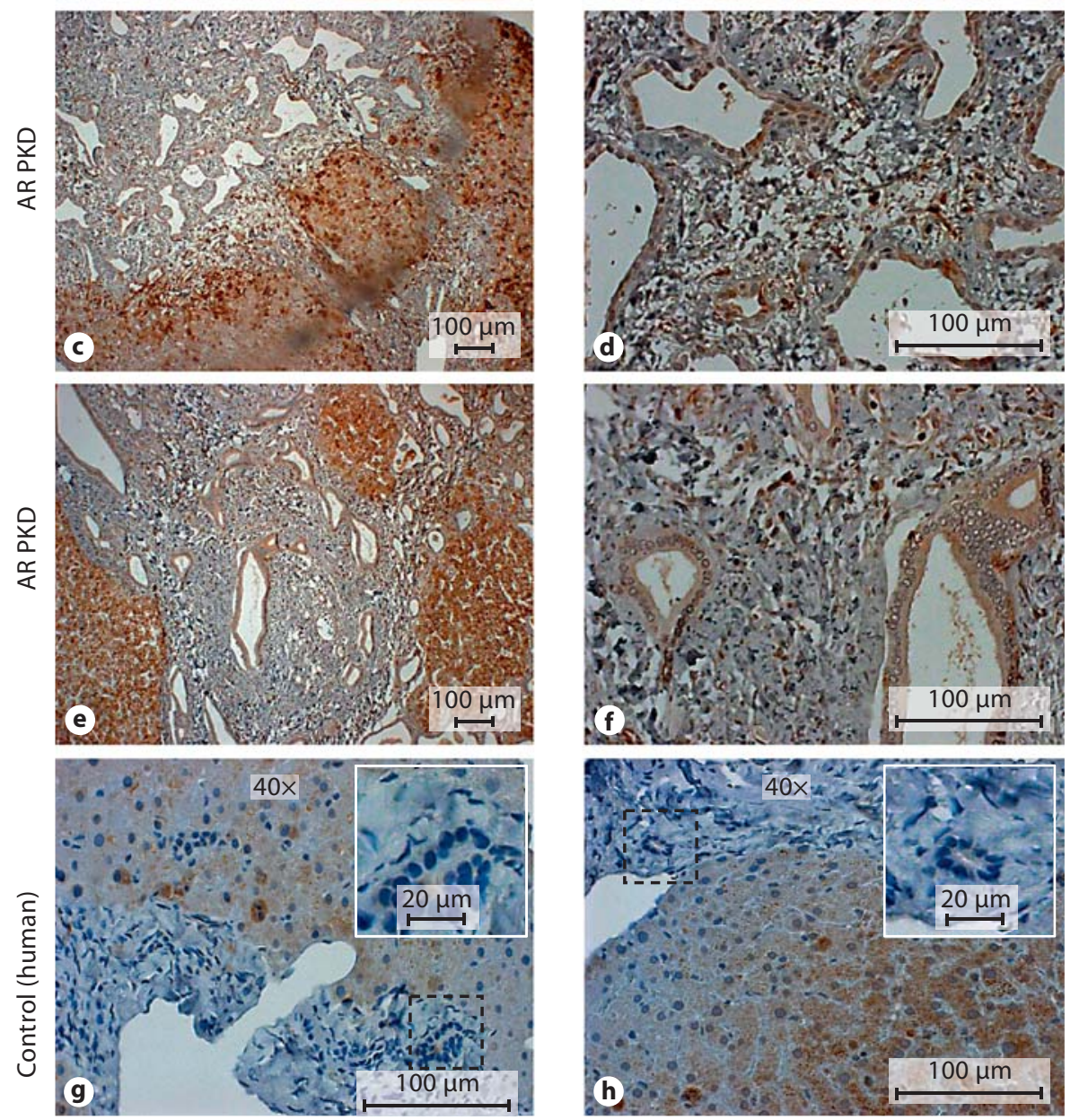

not only regulated by mTOR sensing amino acid sufficiency but is also located in the signaling pathway of growth factors including PDK1 and PKB, two protein kinases that are client proteins of Hsp90 [15]. It may be possible that such complex control mechanisms may account for the different staining pattern observed for pmTOR and pS6K in AR PKD kidneys.
When we analyzed the physiological expression of mTOR molecules in the human kidney, we found strong expression of the phosphorylated molecules pmTOR and pS6K in distal tubule segments. While Shillingford et al. [5] did not show immunostaining of these molecules in the normal human kidney, another group reported renal expression of mTOR-related molecules in 
4E-BP1
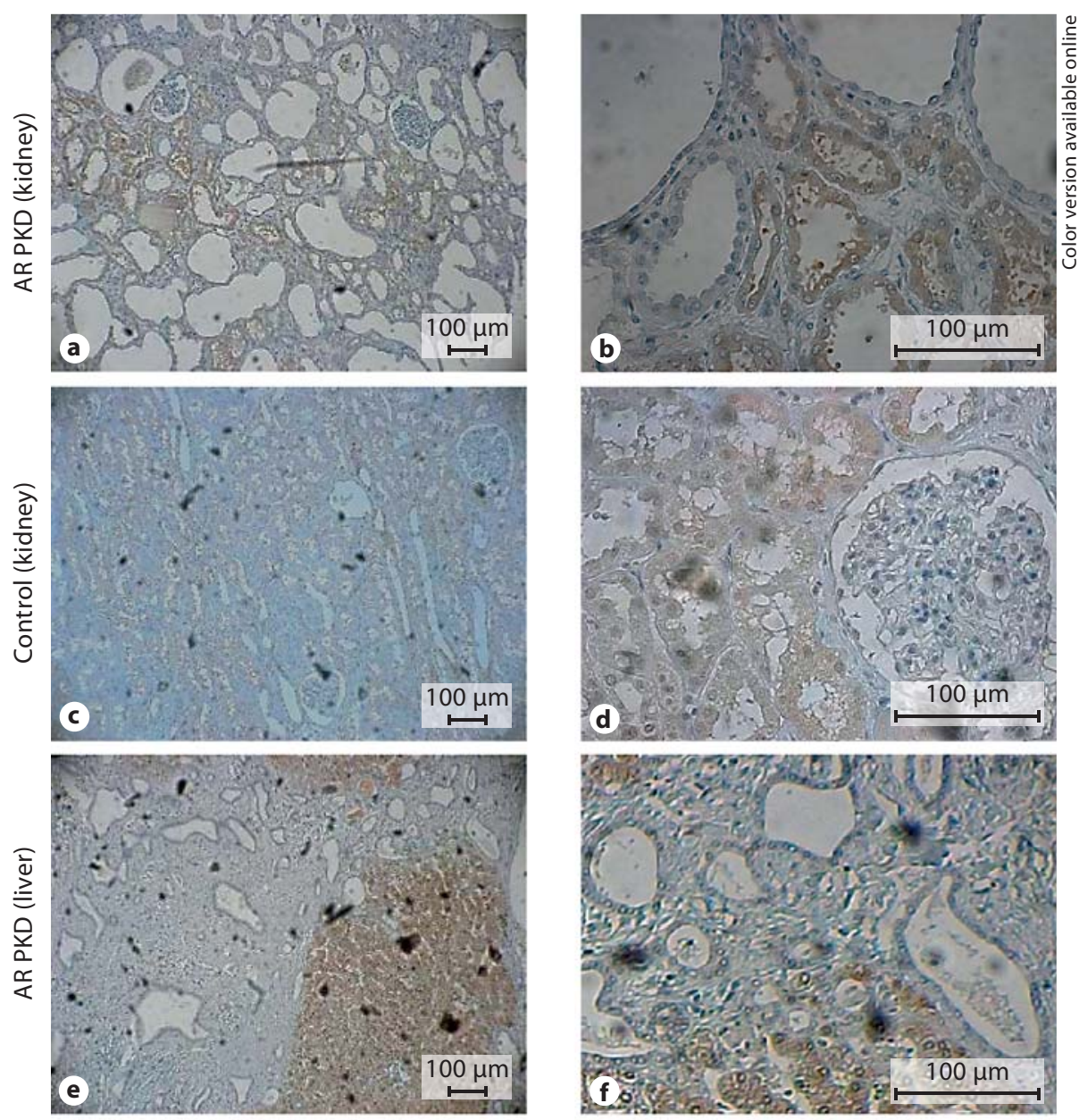

Fig. 5. Renal and hepatic expression of p4E-BP1 and peIF4G. a, c, e, g Lower magnifications at $10 \times \mathbf{b}, \mathbf{d}, \mathbf{f}, \mathbf{h}$ Higher magnifications at $40 \times$ of the same specimens. p4E-BP1 expression in a human AR PKD kidney (a, b), a human control kidney (c, d), and in a human AR PKD liver $(\mathbf{e}, \mathbf{f})$. $\mathbf{g}, \mathbf{h}$ peIF4G expression in an AR PKD (human) liver.
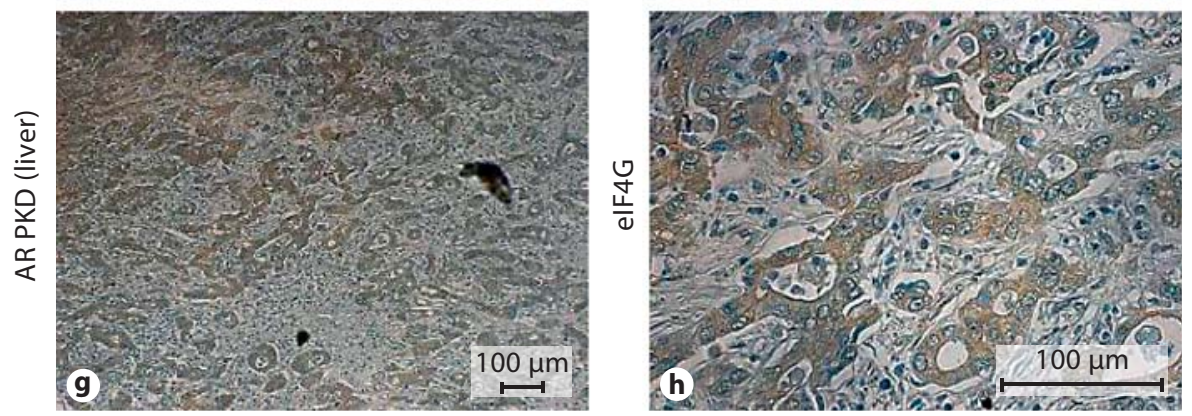

the normal kidney. Consistent with our findings, Kenerson et al. [16] report renal expression of mTOR-related molecules (e.g. pS6K) in distal tubules. To further elucidate the renal expression of mTOR molecules, we immunostained murine and rat kidneys for these molecules, and found consistent results. The physiological significance of expression of these molecules remains currently unclear. However, their constitutive expression in distal tubules and collecting ducts may contribute to the susceptibility for cyst development, which commences in the distal parts of the nephron segment in autosomal recessive forms of human and experimental PKD in rodents [1, 17-19]. 
Liver specimen from AR PKD patients showed a marked granular intense staining for pmTOR molecules in all bile duct cells suggesting that this pathway is activated in the entire bile duct epithelium. This is in line with the observation in the present study that bile ducts in the same AR PKD specimen showed very similar dimensions and morphology. Our observation that all bile ducts are affected in AR PKD is consistent with the clinical course of this biliary disease which almost obligatory manifests as patients age [20].

However, while bile duct morphology showed only little variability in the same specimen, there was a great variability of bile duct morphology between specimens (fig. 2, 4). This is in line with the wide array of clinical phenotypes and disease progression in AR PKD which result from various factors including genetic heterogeneity, modifying and environmental factors [20].

In noncystic tubules in AR PKD kidneys, pmTOR staining was weakly positive in some tubule cells (fig. 1b), suggesting that activation of the mTOR pathway in AR PKD kidneys may successively initiate. Furthermore, pS6K staining in the AR PKD kidney was observed in smaller noncystic tubules. This is consistent with the data provided by Shillingford et al. [5] showing also pS6K expression in smaller tubules. Similarly, p4E-BP1 staining was positive in noncystic tubules in AR PKD kidneys, but no expression was detected in the cystic epithelium. Indeed, after activation and recruitment of mTOR to the translation preinitiation complex (where it phosphorylates $\mathrm{S} 6 \mathrm{~K}$ and $4 \mathrm{E}-\mathrm{BP} 1$ ), $4 \mathrm{E}-\mathrm{BP} 1$ and $\mathrm{S} 6 \mathrm{~K}$ dissociate from this multi-molecule complex [21]. It is possible that this accounts for the differential detection of pmTOR, pS6K, and $\mathrm{p} 4 \mathrm{E}-\mathrm{BP} 1$ in AR PKD kidneys.

We did not find a positive signal for p-tuberin/TSC2 in AR PKD or control kidney or liver specimens. However, activation of these molecules inhibits mTOR activity, and no information concerning the involvement of these upstream pathway molecules could be obtained.

In conclusion, our data indicate that the mTOR pathway is also activated in AR PKD, and that MTOR molecules may thus represent a potential target to slow down cyst development in AR PKD.

\section{Acknowledgements}

Work supported by the Deutsche Forschungsgemeinschaft (J.N., A.K., K.Z., C.B.), the intramural research program of the University Hospital of Essen (IFORES) (J.N., A.K.), the German Kidney Foundation (Deutsche Nierenstiftung) (C.B.), and the START program of the medical faculty of the RWTH Aachen University (C.B.).

\section{References}

1 Wilson PD: Polycystic kidney disease. N Engl J Med 2004;350:151-164.

2 Walz G: Therapeutic approaches in autosomal dominant polycystic kidney disease (ADPKD): is there light at the end of the tunnel? Nephrol Dial Transplant 2006;21:17521757.

3 Inoki K, Corradetti MN, Guan KL: Dysregulation of the TSC-MTOR pathway in human disease. Nat Genet 2005;37:19-24.

4 Wullschleger S, Loewith R, Hall MN: Tor signaling in growth and metabolism. Cell 2006;124:471-484.

5 Shillingford JM, Murcia NS, Larson CH, Low SH, Hedgepeth R, Brown N, Flask CA, Novick AC, Goldfarb DA, Kramer-Zucker A, Walz G, Piontek KB, Germino GG, Weimbs T: The mtor pathway is regulated by polycystin-1, and its inhibition reverses renal cystogenesis in polycystic kidney disease. Proc Natl Acad Sci USA 2006;103: 5466-5471.
6 Harris TE, Lawrence JC Jr: Tor signaling. Sci STKE 2003;2003:re15.

7 Wahl PR, Hir ML, Vogetseder A, Arcaro A, Starke A, Waeckerle-Men Y, Serra AL, Wuthrich RP: Mitotic activation of akt signaling pathway in han:Sprd rats with polycystic kidney disease. Nephrology (Carlton) 2007;12:357-363.

8 Tao Y, Kim J, Schrier RW, Edelstein CL: Rapamycin markedly slows disease progression in a rat model of polycystic kidney disease. J Am Soc Nephrol 2005;16:46-51.

9 Wahl PR, Serra AL, Le Hir M, Molle KD, Hall MN, Wuthrich RP: Inhibition of mtor with sirolimus slows disease progression in han:Sprd rats with autosomal dominant polycystic kidney disease (ADPKD). Nephrol Dial Transplant 2006;21:598-604.
10 Wu M, Wahl PR, Le Hir M, Wackerle-Men Y, Wuthrich RP, Serra AL: Everolimus retards cyst growth and preserves kidney function in a rodent model for polycystic kidney disease. Kidney Blood Press Res 2007;30:253259.

11 Fischer DC, Jacoby U, Pape L, Ward CJ, Kuwertz-Broeking E, Renken C, Nizze H, Querfeld U, Rudolph B, Mueller-Wiefel DE, Bergmann C, Haffner D: Activation of the AKT/MTOR pathway in autosomal recessive polycystic kidney disease (AR PKD). Nephrol Dial Transplant 2009;24:18191827.

12 Edelstein CL: Mammalian target of rapamycin and caspase inhibitors in polycystic kidney disease. Clin J Am Soc Nephrol 2008;3: 1219-1226.

13 Nurnberger J, Kavapurackal R, Zhang SJ, Opazo Saez A, Heusch G, Philipp T, Pietruck F, Kribben A: Differential tissue distribution of the invs gene product inversin. Cell Tissue Res 2006;323:147-155. 
14 Bergmann C, Senderek J, Schneider F, Dornia C, Kupper F, Eggermann T, RudnikSchoneborn S, Kirfel J, Moser M, Buttner R, Zerres K: Pkhd1 mutations in families requesting prenatal diagnosis for autosomal recessive polycystic kidney disease (AR PKD). Human mutation 2004;23:487-495.

15 Avruch J, Belham C, Weng Q, Hara K, Yonezawa K: The p70 s6 kinase integrates nutrient and growth signals to control translational capacity. Prog Mol Subcell Biol 2001;26:115154.

16 Kenerson HL, Aicher LD, True LD, Yeung RS: Activated mammalian target of rapamycin pathway in the pathogenesis of tuberous sclerosis complex renal tumors. Cancer Res 2002;62:5645-5650.
17 Lager DJ, Qian Q, Bengal RJ, Ishibashi M, Torres VE: The pck rat: a new model that resembles human autosomal dominant polycystic kidney and liver disease. Kidney Int 2001;59:126-136.

18 Phillips CL, Miller KJ, Filson AJ, Nurnberger J, Clendenon JL, Cook GW, Dunn KW, Overbeek PA, Gattone VH 2nd, Bacallao RL: Renal cysts of inv/inv mice resemble early infantile nephronophthisis. J Am Soc Nephrol 2004;15:1744-1755.
19 Woollard JR, Punyashtiti R, Richardson S, Masyuk TV, Whelan S, Huang BQ, Lager DJ, van Deursen J, Torres VE, Gattone VH, LaRusso NF, Harris PC, Ward CJ: A mouse model of autosomal recessive polycystic kidney disease with biliary duct and proximal tubule dilatation. Kidney Int 2007;72:328336.

20 Rossetti S, Harris PC: Genotype-phenotype correlations in autosomal dominant and autosomal recessive polycystic kidney disease. J Am Soc Nephrol 2007;18:1374-1380.

21 Holz MK, Ballif BA, Gygi SP, Blenis J: Mtor and s6k1 mediate assembly of the translation preinitiation complex through dynamic protein interchange and ordered phosphorylation events. Cell 2005;123:569-580. 